\title{
OBSERVATIONS OF INTERSTELLAR EXTINCTION \\ IN THE ULTRAVIOLET WITH THE OAO SATELLITE
}

\author{
R. C. BLESS and B. D. SAVAGE \\ Space Astronomy Laboratory, Washburn Observatory, \\ University of Wisconsin, Madison, Wis., U.S.A.
}

\begin{abstract}
We present a preliminary analysis of a number of spectrophotometric scans of reddened and unreddened early-type stars obtained with the OAO-A2 satellite. The principal findings are: (1) all the extinction curves show a 'bump' at $1 / \lambda \approx 4.5 ;(2)$ most extinction curves show a minimum at $1 / \lambda \approx 5.5-6.0 ;(3)$ all the extinction curves show a rapid rise in the far ultraviolet; and (4) there appear to be real differences in the far ultraviolet extinction. A brief comparison of our observed extinction curves is made with theoretical particle models.
\end{abstract}

In this paper we give a preliminary analysis of spectrophotometric scans of a number of early-type stars made over the spectral region 1050-3300 $\AA$ from the OAO-A2 satellite. These scans were made by two objective grating instruments with apertures of $15 \times 20 \mathrm{~cm}$, one sensitive over the interval 1050-1800 $\AA$ with $\sim 15 \AA$ resolution, the second sensitive from $1800-3800 \AA$ with $\sim 20 \AA$ resolution. The gratings, moving in discrete steps about equal to the resolution, sweep the spectrum past a slot behind which is located an Ascop $541 \mathrm{~F}$ photomultiplier for the shorter wavelength instrument and an EM1 6256B photomultiplier for the longer wavelength instrument. With the 8-sec per step integration time used for these scans, $5^{\mathrm{m}}$ early-type stars can be scanned in about $15 \mathrm{~min}$ for each of the two wavelength intervals. Both analog and digital output signals are recorded.

Figure 1 shows representative scans over the 1050-1800 $\AA$ interval of $\sigma$ Ori and $\zeta$ Oph, both $09.5 \mathrm{~V}$ stars, but the latter $0 \mathrm{~m} .28$ redder in $(B-V)$ than the former. Note the strong resonance lines of Si IV and CIV and the blend of stellar Nv, stellar SiIII, and mostly interstellar Lyman $\alpha$. The large number of spectral features makes wavelength identifications and comparisons of two spectra straightforward.

The question of the identity of the ultraviolet spectra of two stars of the same visual color and spectral type is of obvious importance for extinction determinations. Figure 2 gives the results of comparisons of unreddened stars of types B1 II and B3 V. The arrows in the figure indicate the flux ratios for the pairs as obtained from the visual magnitudes. The ultraviolet spectra of these pairs of stars of the same type are remarkably similar. In addition, Figure 2 compares a B1 V and a B1 II star which also have nearly identical continua. Very similar flux ratio plots have been made from several other unreddened pairs having the same spectral types and luminosity classes III and V, and also for early-type unreddened pairs in the $1800-3300 \AA$ region. We may conclude that, unless this admittedly-small sample of stars is atypical, two earlytype stars with the same visual spectral type and of luminosity classes in the range II to $V$ have essentially the same continuous spectrum in the ultraviolet; differences in the continua of two stars of the same spectral type but different colors may therefore be attributed entirely to extinction by the interstellar medium. 


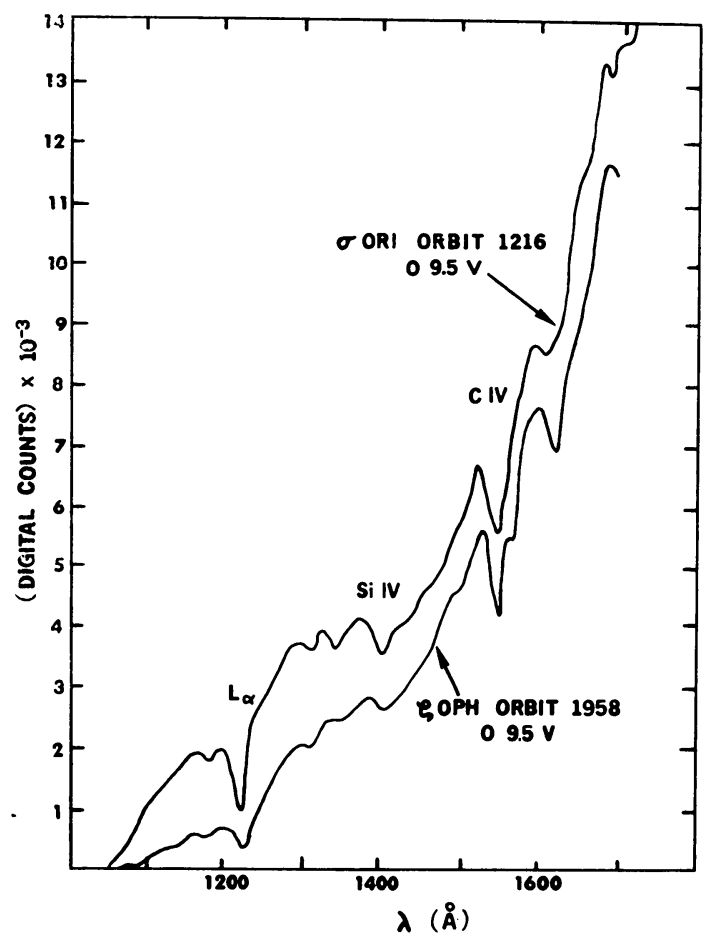

Fig. 1. Spectral scans of $\sigma$ Ori $(B-V=-.24)$ and $\zeta$ Oph $(B-V=+.02)$ made with the short wavelength scanner on OAO-A2. Digital counts for an 8-sec integration time are plotted vs. wavelength (in $\AA$ ). Background counts have been subtracted out.

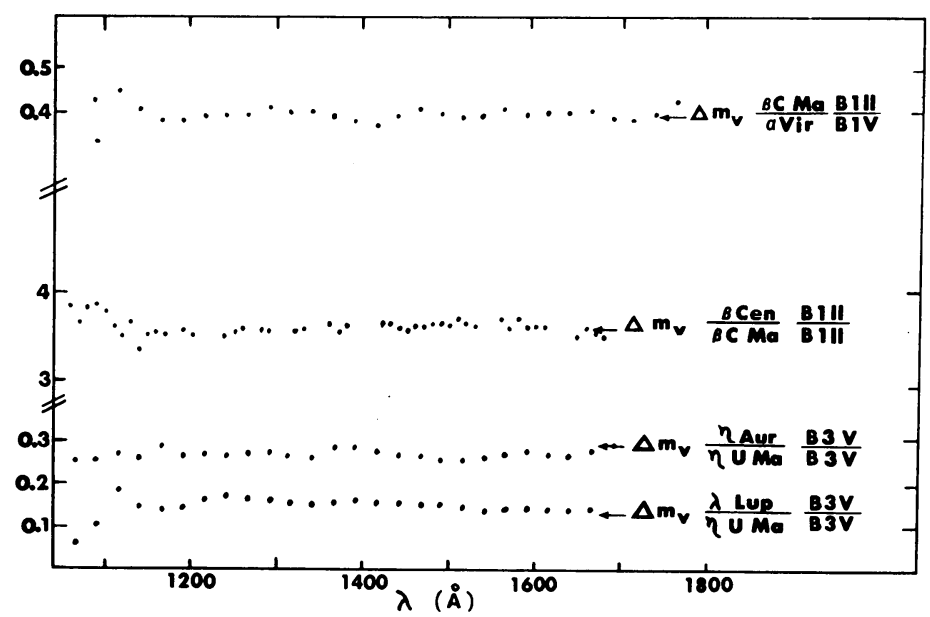

Fig. 2. Flux ratios for a number of pairs of unreddened stars as obtained from the OAO short wavelength scanner data. The $\Delta m_{\mathrm{v}}$ arrows indicate the flux ratios predicted from the difference in the visual magnitudes for each pair. 


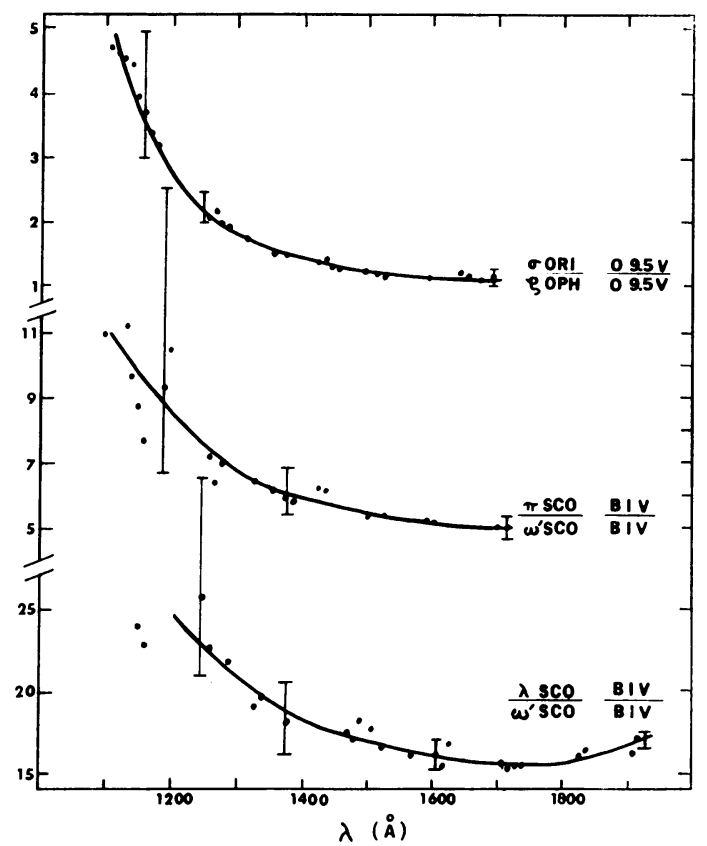

Fig. 3. Flux ratios for three pairs of stars, one unreddened, the other reddened as obtained from the short wavelength scanner data. See Table I for data concerning each star. Error bars indicate generous error estimates. Most of error is due to uncertainty of scattered light background.

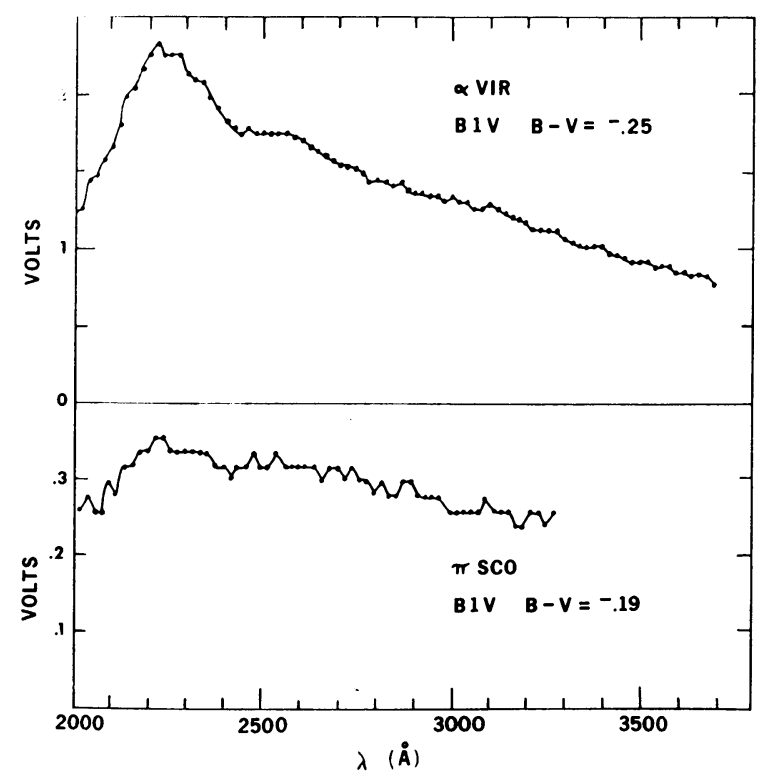

Fig. 4. Spectral scans of $\alpha \operatorname{Vir}(B-V=-.25)$ and $\pi$ Sco $(B-V=-.19)$ made with the long wavelength scanner on OAO-A2. Analog data (volts) are plotted vs. wavelength (in $\AA$ ). 
Point-by-point comparisons of three pairs of stars observed with the short wavelength instrument and with different reddening are given in Figure 3. Flux ratios were computed at points which avoid the strong absorption lines. The error bars shown are generous estimates of the uncertainties caused primarily by possible error in the background count determination. This background, which is chiefly scattered light, is found by observations of objects at wavelengths shortward of the LiF cutoff of the Fabry optics. The background is assumed constant in the 1050-1800 $\AA$ range.

Figure 4 gives two sample scans of a reddened and unreddened star with the longer wavelength instrument. We have plotted analog data although digital data gives better intensity resolution. The spectral resolution is $20 \AA$. Because of the lower resolution and the absence of many strong lines for early $B$ stars it is more difficult to establish a wavelength scale in the 1800-3300 $\AA$ spectral range than in the shorter wavelength

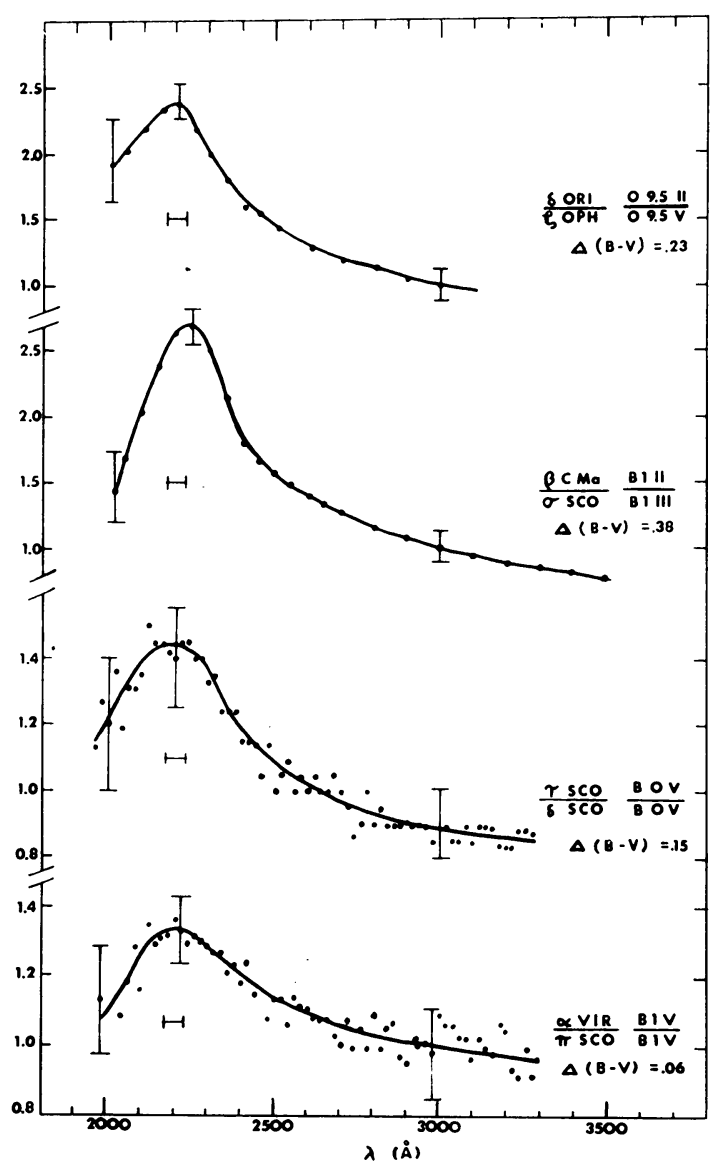

Fig. 5. Flux ratios for stars observed with the long wavelength scanner. In addition to error bars on the ordinate (resulting mainly from an uncertainty in scattered light background) a wavelength determination error bar of $: 30 \AA$ is indicated. All these curves except the $\tau$ Sco- $\delta$ Sco curve are normalized to a flux ratio of 1.0 at $3000 \AA$. 
range. However the $\mathrm{MgII}$ doublet near $2800 \AA$ is usually visible in early B stars and makes the wavelength assignment possible. We have not included a correction for the background radiation in these data for the longer wavelength scanner; it should be possible to do so in the future. Note the considerable difference in the appearance of the two spectra in Figure 4 with a $(B-V)$ difference of only 0 m.06.

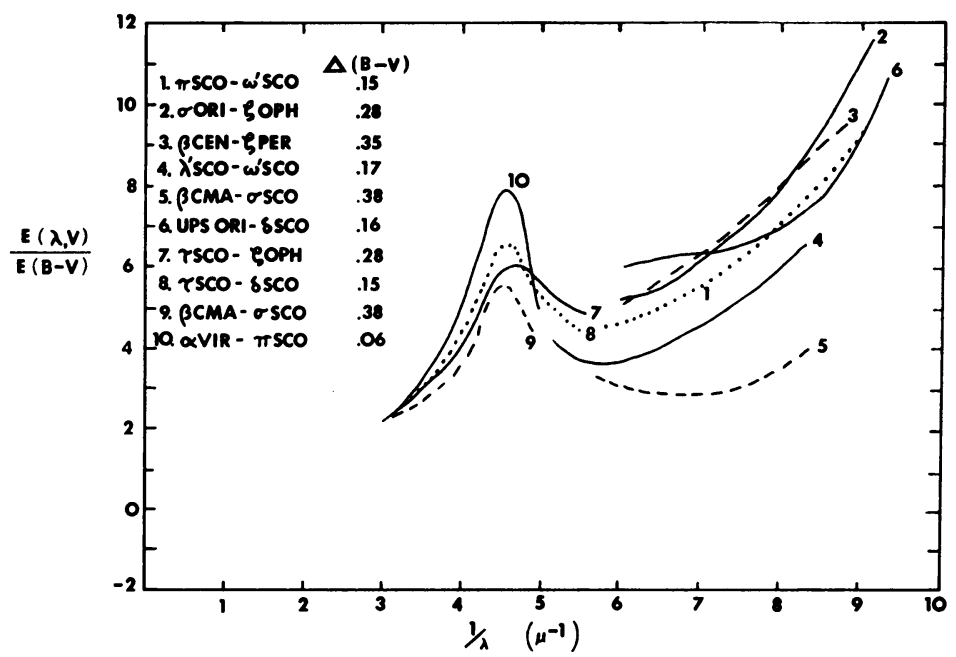

Fig. 6. Preliminary reddening curves for the indicated pairs. For more data concerning the stars see Table I. The curves obtained from the long wavelength scanner data are normalized to $E(3333 \AA-V) / E(B-V)=2.2$. Error bars are not shown but can be easily estimated by referring to

Figures 3 and 5.

\section{TABLE I}

Data on stars used in reddening analysis

\begin{tabular}{lllllll} 
HD & Name & $\begin{array}{l}\text { Spectral } \\
\text { type }\end{array}$ & $V$ & $B-V$ & $\begin{array}{l}\text { Region scanned } \\
(\AA)\end{array}$ & $\begin{array}{l}\text { Orbit of } \\
\text { observation }\end{array}$ \\
\cline { 3 - 6 } & & & & & & \\
24398 & $\zeta$ Per & B1 Ib & 2.83 & +.13 & $1050-1800$ & 892 \\
$36485-86$ & $\delta$ Ori & O9.5 II & 2.20 & -.21 & $1050-3500$ & 955,1247 \\
36512 & $v$ Ori & B0 V & 4.61 & -.27 & $1050-1800$ & 1215 \\
37468 & $\sigma$ Ori & O9.5 V & 3.76 & -.24 & $1050-1800$ & 963,1238 \\
44743 & $\beta$ CMa & B1 II & 1.98 & -.23 & $1050-3500$ & 971 \\
116658 & $\alpha$ Vir & B1 V & 0.97 & -.24 & $1050-3500$ & 795,815 \\
122451 & $\beta$ Cen & B1 II & 0.61 & -.23 & $1050-3500$ & 1802 \\
143018 & $\pi$ Sco & B1 V & 2.90 & -.19 & $1050-3500$ & 1621 \\
143275 & $\delta$ Sco & B0 V & 2.32 & -.11 & $1050-3500$ & 1628 \\
144470 & $\omega 1$ Sco & B1 V & 3.97 & -.04 & $1050-1800$ & 1632 \\
147165 & $\sigma$ Sco & B1 III & 2.89 & +.14 & $1050-3500$ & 1595,1717 \\
149438 & $\tau$ Sco & B0 V & 2.82 & -.26 & $2000-3500$ & 1609 \\
149757 & $\zeta$ Oph & O9.5 V & 2.57 & +.02 & $1050-3500$ & 1704,1955 \\
158926 & $\lambda 1$ Sco & B1 V & 1.62 & -.20 & $1050-1800$ & 2219
\end{tabular}


Flux ratio curves are given in Figure 5 for the longer wavelength scanner. Except for the $\tau$ Sco- $\delta$ Sco pair the flux ratios have been arbitrarily normalized to 1.0 at $3000 \AA$. The marked 'bump' centered at about $2200 \AA$ seems to be a characteristic feature of the interstellar extinction.

We have compiled several extinction curves from comparison of pairs of stars observed with the two OAO scanners. The reddened stars are in Orion, Scorpius, Ophiuchus, and Perseus. These curves are given in Figure 6 where the ratio of $(\lambda-V)$ excess to $(B-V)$ excess for the longer wavelength scanner comparisons is adjusted to 2.2 at $1 / \lambda=3$, the mean value found by Boggess and Borgman (1964). Data concerning the stars used for the extinction determinations are given in Table $I$. It is evident that a slight error in $(B-V)$ can cause a considerable shift of an extinction curve along the ordinate. For example, the difference between the extinction curves for the comparison pairs 1 and 4 is likely due to the 10 to $20 \%$ uncertainty in the $\Delta(B-V)$ for these pairs. Thus the shape of the curves is the more significant feature, not the vertical position.

Several comments can be made about these extinction curves:

(1) All show the 'bump' at $1 / \lambda \approx 4.5$.

(2) Most show a minimum at $1 / \lambda \approx 5.5-6.0$ but curves 5 and possibly 6 deviate significantly from the shape of the others, curve 5 having a minimum at $1 / \lambda \approx 7.0$.

(3) All show a rapid rise in extinction in the far ultraviolet.

(4) Apparently there are real differences in the far UV extinction.*

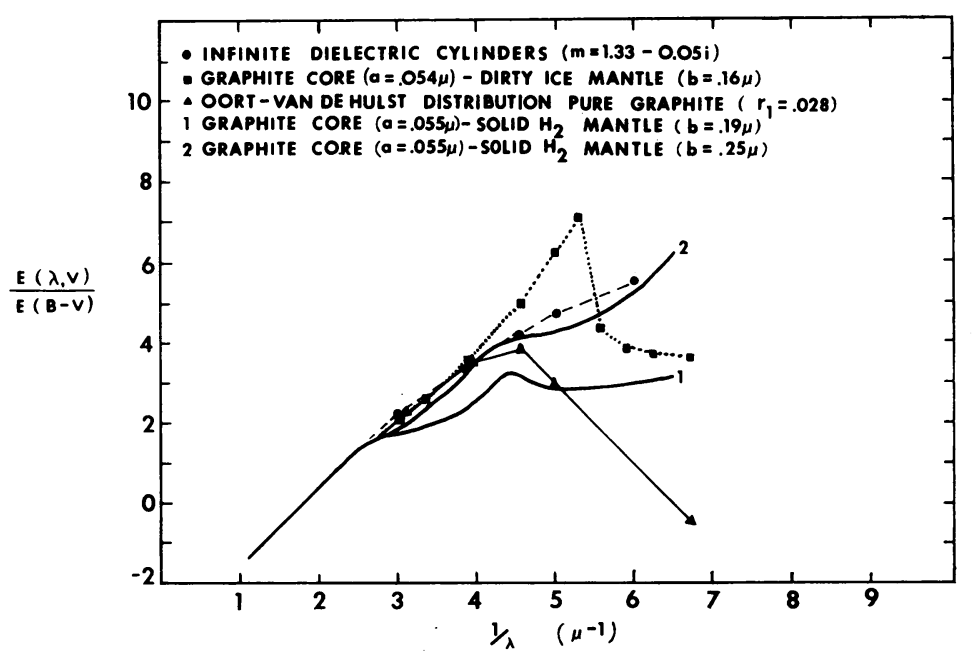

Fig. 7. Shown are a number of theoretical reddening curves: infinite dielectric cylinders of Greenberg and Shah (1969); $\square$ graphite-core and dirty ice mantle of Greenberg and Shah (1969); $\Delta$ Oort-Van de Hulst distribution of pure graphite from Krishna Swamy and O'Dell (1967); and 1,2 graphite core- $\mathrm{H}_{2}$ mantle from Wickramasinghe and Nandy (1968). $m=$ dielectric constant, $a=$ core radius, $b=$ mantle radius.

* For OAO results near the Trapezium see on p. 107 the comment by Bless in the discussion following Carruthers' paper. 


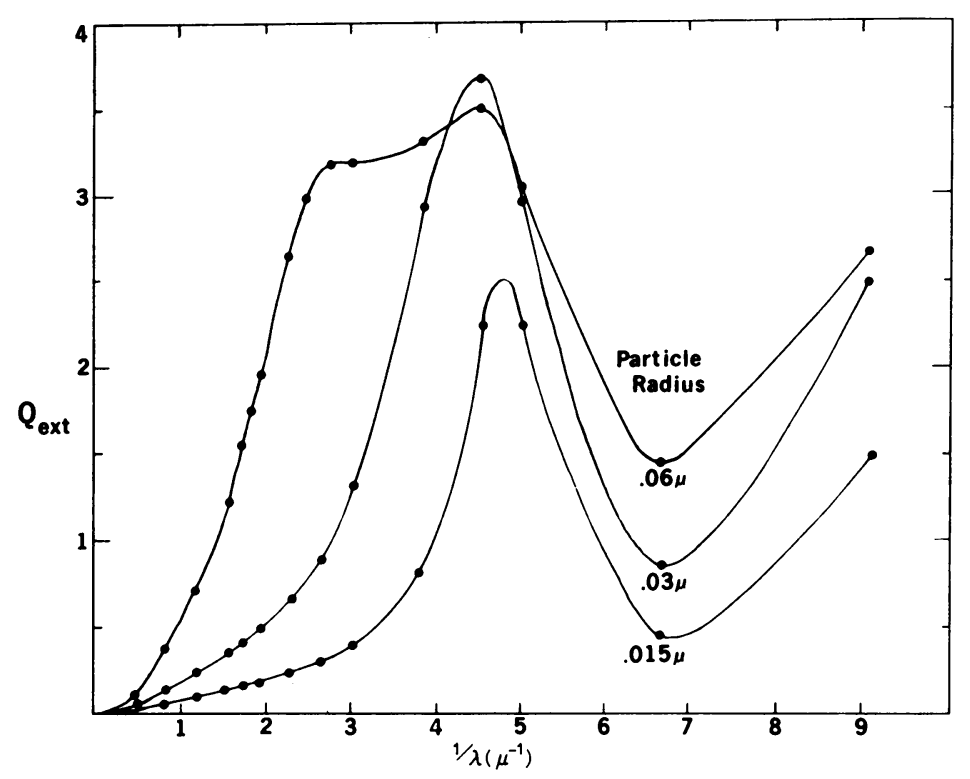

Fig. 8. Qextinction for various sizes of pure graphite particles from Grevesse-Guillaume and Wickramasinghe (1966). Note that the peak in extinction for small graphite particles occurs at $1 / \lambda \sim 4.5$.

The extinction curve adopted by Bless et al. (1968) from various filter photometry measurements shows the same general trend as those given here, but both the "bump' and the minima found in the $\mathrm{OAO}$ results were smoothed over.

Finally, we would like to compare these results with predictions of a few of the models of interstellar particles recently published and shown in Figure 7. The infinite dielectric cylinder model calculated by Greenberg and Shah (1969) shows no 'bump' at $2200 \AA$. The graphite core with a dirty ice mantle considered by Greenberg and Shah (1969) has an extinction 'bump' at shorter wavelengths to a degree inconsistent with these observations. The extinction by an Oort-Van de Hulst distribution of sizes of pure graphite particles (Krishna Swamy and O'Dell, 1967) gives a peak at the correct wavelength but does not give the far ultraviolet extinction increase. One must be careful in interpreting the numerous extinction calculations for pure graphite, however, because some results are based on old inaccurate models of the electrical properties of graphite (Krishna Swamy and Wickramasinghe, 1966) while others are based on laboratory measurements of these electrical properties (Grevesse-Guillaume and Wickramasinghe, 1966). Several extinction curves from the paper of Grevesse-Guillaume and Wickramasinghe (1966) are shown in Figure 8. One can see that pure graphite particles of small size display the 'bump' observed in the OAO data. The particle model with a rather large graphite core coated with a $\mathrm{H}_{2}$ mantle (Wickramasinghe and Nandy, 1968) retains the $2200 \AA$ 'bump' characteristic of graphite if the mantle is thin (see Figure 7) and also shows the far UV extinction increase. The 'bump' is not as pronounced as the observations indicate, however. 
We must emphasize that agreement or disagreement between the observations and any of the particle models given above is relevant only for that particular model; changes in the parameters of a particle type can produce marked changes in its extinction characteristics. In all probability interstellar particles have a variety of sizes, shapes, and compositions whose parameters may be modified by local conditions.

A considerable amount of OAO scanner data remains to be analyzed as well as all of the filter photometry of reddened stars. This large body of extinction data may, hopefully, set sharper limits to the properties of interstellar grains than has heretofore been possible.

\section{References}

Bless, R. C., Code, A. D., and Houck, T. E.: 1968, Astrophys. J. 153, 561.

Boggess, A. and Borgman, J.: 1964, Astrophys. J. 140, 1636.

Greenberg, J. M. and Shah, G.: 1969, Physica 41, 92.

Grevesse-Guillaume, C. and Wickramasinghe, N. C.: 1966, Monthly Notices Roy. Astron. Soc. 132, 471.

Krishna Swamy, K. S. and O’Dell, C. R.: 1967, Astrophys. J. 147, 937.

Krishna Swamy, K. S. and Wickramasinghe, N. C.: 1966, Monthly Notices Roy. Astron. Soc. $132,193$. Wickramasinghe, N. C. and Nandy, K.: 1968, Nature 219, 1347.

\section{Discussion}

Wickramasinghe: You pointed out that pure graphite has an absorption feature at $2200 \AA$. It turns out that the band continues to persist in highly impure graphite as well. It has been observed in soots as well as in charcoal.

Bless: We don't insist on pure graphite, but simply point out that it is one of the particle types which could account for the bump.

Wilson: With regard to the theoretical extinction curves you showed for the two composite particles, each with a graphite core but one with an ice mantle and the other with solid hydrogen, I noted that the feature near $2200 \AA$ was much more pronounced in one than in the other. Since the feature is supposed to be caused by the graphite, which in each case is surrounded by a dielectric mantle, I find it rather surprising. Can you offer a physical explanation?

Bless: Any thick mantle on a graphite core will smooth out the $2200 \AA$ feature. If graphite is responsible for the bump then at least some of the particles must have thin mantles or no mantle at all.

Van de Hulst: Do your observations agree with those by Stecher?

Bless: Yes, the main features agree well. 